\title{
A NEW EXTENSION OF CARLSON'S INEQUALITY
}

\author{
Min-Jie Luo AND RAVINDER KRISHna RAinA
}

Abstract. A new extension of Carlson's inequality is obtained by using the Euler-Maclaurin summation formula and an extended Beta function introduced recently in [Appl. Math. Comput. 248 (2014) 631-651]. The integral analogue of this inequality is also presented.

Mathematics subject classification (2010): 26D15, 33C15.

Keywords and phrases: Carlson's inequality, Euler-Maclaurin summation formula, extended Beta function.

\section{REFERENCES}

[1] T. M. Apostol, An elementary view of Euler's summation formula, Amer. Math. Monthly 106 (1999), 409-418.

[2] S. BArZA, J. PeČArić, L.-E. Persson, Carlson type inequalities, J. Inequal. Appl. 2 (1998), 121135.

[3] F. CARLson, Une inegalité, Ark. Mat. Astr. Fysik, 25B (1934), 1-5.

[4] G. H. HARDY, A note on two inequalities, J. London Math. Soc., 11 (1936), 167-170.

[5] K. Jichang, L. Debnath, An extension of Carlson's inequality, J. Math. Anal. Appl. 267 (1) (2002), 395-399.

[6] L. Larsson, L. Maligranda, J. PeČArić, L.-E. Persson, Multiplicative Inequalities of Carlson Type and Interpolation, World Scientific, New Jersey, London, Singapore, Beijing, Shanghai, Hong Kong, Taipei, and Chennai, 2006.

[7] Min-Jie Luo, G. V. Milovanovic, P. Agarwal, Some results on the extended beta and extended hypergeometric functions, Appl. Math. Comput. 248 (2014), 631-651.

[8] H. M. SRivastava, J. Choi, Zeta and q-Zeta Functions and Associated Series and Integrals, Elsevier Science, Publishers, Amsterdam, London and New York, 2012.

[9] H. M. Srivastava, H. L. Manocha, A Treatise on Generating Functions, Halsted Press (Ellis Horwood Limited, Chichester), John Wiley and Sons, New York, Chichester, Brisbane, and Toronto, 1984. 